\title{
A depressão pós-parto em mulheres que sobreviveram à morbidade materna grave
}

Postpartum depression in women who survived severe maternal morbidity

\author{
Mônica Silva Silveira', Ricardo Queiroz Gurgel ${ }^{1}$, Íkaro Daniel de Carvalho Barreto", \\ Leda Maria Delmondes Freitas Trindade ${ }^{2}$
}

\begin{abstract}
Resumo
Introdução: A morbidade materna grave é cada vez mais conhecida como um indicador útil de segurança e de qualidade do cuidado materno e pode afetar a saúde mental da mãe. Objetivo: Avaliar a relação entre a morbidade materna grave (near miss) e os sintomas da depressão pós-parto. Método: Estudo descritivo de coorte prospectivo. A amostra foi constituída por 549 mulheres puérperas em duas maternidades públicas do Estado de Sergipe. Foi aplicada a Edinburgh Postnatal Depression Scale (EPDS) para identificar os sintomas de depressão no pós-natal. Para a análise estatística, aplicaram-se os testes do Qui-quadrado, de U-Mann-Whitney e o coeficiente de correlação de postos de Spearman, e considerou-se o nível de significância de $\alpha \leq 0,05 \%$. Resultados: 156 (56\%) das mães expostas à MMG/NM e 45 (17\%) das não expostas revelaram forte associação com depressão pós-parto e maior chance (ORC: 24,0; IC95\%: 7,23-79,7) de desenvolvê-la. Conclusão: A MMG/NM tem impacto negativo na saúde mental da mulher e eleva a sua vulnerabilidade para a doença mental. É fundamental para a qualidade da assistência materno-infantil a implantação de políticas públicas que assegurem prevenção e estratégias de enfrentamento.

Palavras-chave: morbidade; near miss; saúde materna; depressão; depressão pós-parto.
\end{abstract}

\begin{abstract}
Background: Severe maternal morbidity is increasingly known as a useful indicator of the safety and quality of maternal care, and may affect the mother's mental health. Objective: To evaluate the relationship between severe maternal morbidity (near miss) and symptoms of postpartum depression. Method: Descriptive study of prospective cohort. The sample consisted of a total of 549 puerperal women from two public maternity hospitals in the state of Sergipe. We applied the Edinburgh Postnatal Depression Scale (EPDS) to identify the prevalence of postpartum depression. The statistical analysis was performed using chi-square test of U-Mann-Whitney and the Spearman rank correlation coefficient, with significance level of $\alpha \leq 0.05 \%$. Results: 156 (56\%) of the mothers exposed to severe maternal obesity, and $45(17 \%)$ of the non-exposed mothers showed a strong association and greater chance (ORC: $24.0 ; 95 \% \mathrm{Cl}: 7.23-79.7)$ to develop postpartum depression. Conclusion: Severe maternal morbidity has a negative impact on women's mental health, and increases the vulnerability to mental illness. It is fundamental for the quality of maternal and child care to implement public policies that ensure prevention and coping strategies.
\end{abstract}

Keywords: morbidity; near miss; maternal health; depression; postpartum depression.

1Programa de Pós-graduação e Pesquisa em Ciências da Saúde (PPGCS), Universidade Federal de Sergipe (UFS) - Aracaju (SE), Brasil.

²Departamento de Medicina, Universidade Federal de Sergipe (UFS) - Aracaju (SE), Brasil.

Trabalho realizado na Maternidade Nossa Senhora de Lourdes e no Hospital e Maternidade Santa Isabel - Aracaju (SE), Brasil.

Endereço para correspondência: Mônica Silva Silveira - Rua João Gêniton da Costa, 400, Bloco 02, ap. 03, térreo, Condomínio Natura Ville - Bairro Jabotiana CEP: 49095-796 - Aracaju (SE), Brasil - Email: mssdoutora@gmail.com

Fonte de financiamento: nenhuma.

Conflito de interesses: nada a declarar. 


\section{INTRODUÇÃO}

A morbidade materna grave é cada vez mais conhecida como um indicador útil de segurança e qualidade do cuidado materno, por isso o conhecimento dos seus fatores de risco e de suas consequências pode contribuir para a definição de políticas públicas e estratégias na saúde integral. Apesar do número estimado de mulheres que sofrem de complicações maternas, pouco se sabe sobre os seus efeitos na saúde mental ${ }^{1,2}$.

A morbidade materna grave, também conhecida como near miss, é um evento de quase morte causado por complicações graves ocorridas com a mulher durante a gravidez, parto ou puerpério ${ }^{3}$. Os critérios desse diagnóstico representam identificadores clínicos, laboratoriais e de gerenciamento aplicáveis tanto em países desenvolvidos quanto em desenvolvimento ${ }^{4}$.

Segundo a Organização Mundial de Saúde (OMS), os diagnósticos de morbidade materna grave e near miss (MMG/NM) podem incluir várias patologias, tais como distúrbios hemorrágicos, distúrbios hipertensivos e outras doenças sistêmicas, edema pulmonar, insuficiência respiratória e convulsão durante a gravidez, além de indicadores de cuidados críticos, de transfusão de sangue, histerectomia e unidade de terapia intensiva ${ }^{4}$.

Conforme Furruta et al. ${ }^{1}$, a morbidade materna grave causa prejuízos psicológicos e sociais a longo prazo para a mulher e toda a família. Considera-se que a morbidade materna grave pode repercutir no estado emocional da mulher em diversos aspectos, além de afetar negativamente a sua qualidade de vida por um longo período após o evento ${ }^{5,6}$. De acordo com a OMS, cerca de $10 \%$ das mulheres grávidas e $13 \%$ das puérperas apresentam um transtorno mental, e a depressão pós-parto pode atingir de 10 a $20 \%$ das puérperas ${ }^{7}$.

Segundo a American Psychiatric Association ${ }^{8}, 50 \%$ dos episódios de depressão que ocorrem no puerpério se desenvolveram no início da gravidez. Por essa razão, o especificador "com início no pós-parto" para os transtornos depressivos e bipolares foi substituído para "com início periparto". Os sintomas assemelham-se aos transtornos depressivos existentes em outros períodos da vida, além de poder afetar a relação e a qualidade da interação entre a díade mãe-filho9.

Entre os fatores significativos que contribuem para o surgimento da depressão pós-parto, incluem-se depressão pré-natal, ansiedade, história psiquiátrica pregressa, relacionamento conjugal conflituoso, eventos estressantes, atitude negativa em relação à gravidez e falta de apoio social ${ }^{10}$.

Diante do exposto, por se tratar de um tema recente, pouco se sabe sobre as repercussões da morbidade materna grave na saúde mental das mães. Este estudo tem por objetivo avaliar a relação entre a morbidade materna grave $(\mathrm{MMG} / \mathrm{NM})$ e os sintomas da depressão pós-parto.

\section{MÉTODO}

Estudo de coorte prospectivo, constituído por puérperas das duas maternidades de referência para médio e alto risco obstétrico do Sistema Único de Saúde (SUS) do Estado de Sergipe, Nordeste do Brasil. A coleta de dados foi realizada de janeiro a maio do ano de 2013.

Para a seleção da amostra dos casos elegíveis, foi realizada, a cada 48 horas, a busca ativa nas duas maternidades de referência do Estado de Sergipe, por meio do livro de registro de internação hospitalar das enfermarias de obstetrícia, do puerpério e da análise dos prontuários, a fim de que pudessem ser identificadas e entrevistadas as puérperas. Não houve perda durante a coleta de dados.

O total da amostra foi de 549 sujeitos, constituídos em dois grupos: o grupo de risco, com 277 mulheres expostas à morbidade materna grave e near miss, que preenchiam os critérios atuais de diagnóstico, de acordo com a Organização Mundial da Saúde ; e o grupo controle, com 272 puérperas não expostas, sem intercorrência de complicação obstétrica relacionada ao mesmo quadro.

Considerou-se como critérios de exclusão, independentemente do grupo, as puérperas que não residiam no Estado de Sergipe, que tiveram bebês com grave deficiência, que ficaram hospitalizados ou foram a óbito, e as puérperas com restrição médica devido ao seu estado de gravidade. Justificam-se as últimas exclusões pelo fato de que essas situações já provocam o comprometimento do estado emocional, dificultando relacioná-lo à situação de morbidade materna grave e near miss, além de agir como variável de confundimento.

Foram aplicados dois instrumentos por um psicólogo e pesquisador devidamente habilitados: um questionário para avaliar o perfil sociodemográfico, composto pelas variáveis procedência da residência fixa (capital ou interior), idade, escolaridade, situação conjugal, primípara, acompanhamento do pré-natal, trabalho remunerado, uso de álcool, tabagismo e as características clínico-obstétricas; e outro para identificar sintomas de depressão no pós-natal por meio da Escala de Edinburgh Postnatal Depression Scale (EPDS), que consiste em uma escala de autopreenchimento.

A EPDS é composta por dez itens que recebem a pontuação de 0 a 3, de acordo com a intensidade relatada do sintoma depressivo no pós-natal ${ }^{11}$. A pontuação total da escala varia de 0 a 30 pontos. No Brasil, foi validada e traduzida por Santos et al. ${ }^{12}$ Os autores sugeriram ponto de coorte de 11/12, valor utilizado no presente estudo ${ }^{12}$.

Para delimitação do cálculo amostral, considerou-se um tamanho de efeito de 0,14 , significância de $5 \%$ e poder de $90 \%$, levando a uma amostra de 536 indivíduos. Foram entrevistados 549 indivíduos, o que levou a um tamanho de efeito de 0,138 , muito próximo ao estipulado inicialmente. 
Os dados foram analisados pelo software R Core Team 2017, com aplicação das frequências simples e percentual, das médias, do desvio-padrão das variáveis, do teste do Qui-quadrado de Pearson para associação entre variáveis categóricas, do teste de U-Mann-Whitney (média) para identificar as diferenças de média entre os grupos e do coeficiente de correlação de postos de Spearman (Rho). Adotou-se nível de significância de $\alpha \leq 0,05 \%$.

Todas as puérperas, após aceitarem participar da pesquisa, assinaram o Termo de Consentimento Livre e Esclarecido (TCLE) segundo Resolução no 466/2012 do Conselho Nacional de Saúde. O estudo foi aprovado pelo Comitê de Ética em Pesquisa
Envolvendo Seres Humanos da Universidade de Federal de Sergipe (Processo no 0284.1.107.000/2011).

\section{RESULTADOS}

Do total da amostra, foram incluídas 549 participantes. Quanto às características sociodemográficas, clínico-obstétricas e hábitos sociais (álcool e tabagismo) (Tabela 1), foi observado que 182 (66\%) puérperas expostas à MMG/NM eram provenientes do interior do Estado de Sergipe, sendo estatisticamente significativo $(\mathrm{p}<0,001)$.

Os grupos estudados de expostas e não expostas à MMG/NM apresentaram faixa etária abaixo de 35 anos, escolaridade abaixo

Tabela 1. Caracterização sociodemográfica, clínica, obstétrica e hábitos da amostra de mulheres com e sem morbidade materna grave/near miss (MMG/NM), Sergipe, Brasil, 2013

\begin{tabular}{|c|c|c|c|}
\hline \multirow{3}{*}{ Variáveis } & \multicolumn{2}{|c|}{ Grupos } & \multirow{3}{*}{ p-valor } \\
\hline & Com MMG/NM $(n=277)$ & Sem MMG/NM $(n=272)$ & \\
\hline & n (\%) & n (\%) & \\
\hline \multicolumn{4}{|l|}{ Procedência } \\
\hline Capital & $95(34)$ & $200(74)$ & \multirow[t]{2}{*}{0,046} \\
\hline Interior & $182(66)$ & $72(26)$ & \\
\hline Idade média (DP) & $28,15(7,75)$ & $26,77(7,75)$ & $0,038^{\star}$ \\
\hline$\geq 35$ & $70(25)$ & $53(20)$ & 0,104 \\
\hline$<35$ & $207(75)$ & $219(80)$ & \\
\hline \multicolumn{4}{|l|}{ Escolaridade } \\
\hline$<8$ anos & $198(53,5)$ & $172(46,5)$ & \multirow[t]{2}{*}{0,039} \\
\hline$\geq 8$ anos & $79(44,1)$ & $100(55,9)$ & \\
\hline \multicolumn{4}{|l|}{ Situação conjugal } \\
\hline Solteira & $59(21)$ & $75(28)$ & \multirow[t]{2}{*}{0,087} \\
\hline Casada & $218(79)$ & $197(72)$ & \\
\hline \multicolumn{4}{|l|}{ Trabalho } \\
\hline Não & $222(80)$ & $185(68)$ & \multirow[t]{2}{*}{$<0,001$} \\
\hline Sim & $55(20)$ & $87(32)$ & \\
\hline \multicolumn{4}{|l|}{ Pré-natal } \\
\hline Não & $26(9)$ & $3(1)$ & \multirow[t]{2}{*}{$<0,001$} \\
\hline Sim & $251(91)$ & 269 (99) & \\
\hline \multicolumn{4}{|l|}{ Paridade } \\
\hline Primípara & $43(16)$ & $32(12)$ & \multirow[t]{2}{*}{0,200} \\
\hline 1 ou mais parto & $234(84)$ & $240(88)$ & \\
\hline \multicolumn{4}{|l|}{ Bebida alcoólica } \\
\hline Não & 192(69) & $243(89)$ & \multirow[t]{2}{*}{$<0,001$} \\
\hline Sim & $85(31)$ & $29(11)$ & \\
\hline \multicolumn{4}{|l|}{ Tabagismo } \\
\hline Não & $261(94)$ & $263(97)$ & \multirow[t]{2}{*}{0,166} \\
\hline Sim & $16(6)$ & $9(3)$ & \\
\hline
\end{tabular}

Teste Qui-Quadrado de Pearson; ${ }^{\star}$ Teste de Mann-Whitney; DP - Desvio Padrão 
de oito anos e não tinham atividade laboral remunerada. Entre as puérperas expostas à MMG/NM, foi estatisticamente significativo $(\mathrm{p}<0,001)$ mulheres que não fizeram acompanhamento do pré-natal e mulheres que fizeram uso de bebida alcoólica durante a gestação.

Utilizando a Edinburgh Postnatal Depression Scale (EPDS) (Tabela 2), identificou-se que 156 (56\%) expostas e 45 (17\%) não expostas sinalizaram sintomas de depressão pós-natal (OR: 6,5; IC95\%: 4,37-9,69). A análise a partir da regressão logística da amostra de expostas e não expostas à MMG/NM, referente à depressão pós-parto, observou uma forte associação (ORC: 24,0; IC95\%: 7,23-79,7) com a depressão no pós-parto no grupo de expostas à MMG/NM.

Tabela 2. Associação logística da amostra de mulheres com e sem morbidade materna grave/near miss (MMG/NM), referente à depressão pós-parto (Edinburgh Postnatal Depression Scale, EPDS), Sergipe, Brasil. 2013

\begin{tabular}{|c|c|c|c|c|c|}
\hline \multirow{2}{*}{ Variáveis } & \multicolumn{2}{|c|}{ Grupos } & \multirow{2}{*}{ p-valor } & \multirow{2}{*}{ OR (IC95\%) } & \multirow{2}{*}{ ORC (IC95\%) } \\
\hline & Com MMG/NM n (\%) & Sem MMG/NM n (\%) & & & \\
\hline \multicolumn{6}{|l|}{ EPDS } \\
\hline$\geq 12$ & $156(56)$ & $45(17)$ & $<0,001$ & $6,5(4,37-9,69)$ & $24,0(7,23-79,7)$ \\
\hline$<12$ & $121(44)$ & $227(83)$ & & & \\
\hline
\end{tabular}

$\mathrm{OR}=$ odds ratio $; \mathrm{ORC}=$ odds ratio controlada para variáveis demográficas; IC95\% = intervalo de confiança $(95 \%) .(\mathrm{p}<0,05)$

\section{DISCUSSÃO}

No presente estudo, a maioria das mulheres expostas à morbidade materna grave e near miss, em comparação às não expostas, era advinda de cidades afastadas dos centros urbanos, não teve assistência de pré-natal e tinha o hábito de consumir bebida alcoólica e tabaco. O perfil das mulheres com morbidade materna grave e near miss encontrado neste estudo é concordante com o de outras pesquisas ${ }^{13-16}$.

Pode-se observar que foram encontrados maior presença de sintomas da depressão pós-parto no grupo de expostas à morbidade materna grave e near miss e elevado risco de chance do seu desenvolvimento, em comparação ao grupo de não expostas. Pressupõe-se que a condição clínica da morbidade materna grave e near miss possa aumentar sobremaneira a chance desenvolvimento de depressão pós-parto. A mulher que apresenta o evento da morbidade materna grave pode ser acometida pela depressão pós-natal. Segundo Filippi et al. ${ }^{17}$, as mulheres com complicações obstétricas graves foram significativamente mais propensas a ter depressão e ansiedade do que as mulheres com parto não complicado, constatando-se o efeito negativo da morbidade materna em suas vidas em todos os momentos da gravidez até o pós-natal.

Em uma revisão sistemática da saúde mental perinatal na África, foram relatadas taxas de prevalência da depressão na gravidez de 11,3 a 18,3\% no pós-natal. Os fatores associados mais citados referentes à piora da saúde mental foram as complicações obstétricas, a falta de apoio e os conflitos conjugal e familiar ${ }^{18,19}$.

Estudo de coorte prospectivo realizado por Kirti Yyengar et al. ${ }^{2}$, com uma população de puérperas que apresentou complicação obstétrica grave durante e após o parto, indicou a prevalência de depressão pós-parto em torno de 33,3\% e de 18,5\% em mulheres sem complicações obstétricas. Os autores evidenciaram inúmeras dificuldades em função dessa complicação, tais como problemas sociais e financeiros, devido ao afastamento do mercado de trabalho e ao alto custo do tratamento médico.

Diversos estudos revelaram uma associação entre sintomas depressivos pós-parto e as complicações na gravidez e no parto. Para Burgut et al. ${ }^{19}$, em estudo realizado no Oriente Médio, essa associação teve uma prevalência de 17,6\%. Na Argentina, Mathisen et al. ${ }^{20}$ constataram 37,2\% dessa associação entre as mulheres puérperas. Outro dado importante sobre essa associação observado por Norhayati et al. ${ }^{10}$ foi a presença de fatores de risco relacionados a problemas de saúde, complicações obstétricas e baixo apoio social ${ }^{21}$.

Os fatores pré-natais, perinatais e pós-natais causam consequências negativas, podendo levar a óbito materno e infantil ou causar morbidades e deficiências durante o período pós-parto prolongado, em média de até um ano, que impactam negativamente na saúde do bebê, na saúde de outros filhos e na posição social e econômica da família ${ }^{6}$. Ou seja, o evento da morbidade materna grave e near miss na vida dessas mulheres pode repercutir mesmo após resolução do processo da doença.

As situações de gravidade, como sobrevivência da morbidade materna grave, atinge três dimensões que interagem entre si: a primeira diz respeito à ruptura da integridade física por meio das lesões, doença permanente e perda da força e resistência; a segunda abrange a economia doméstica por intermédio dos gastos com problemas de saúde e a perda da capacidade produtiva; a terceira abrange a interrupção da vida social, relacional/conjugal e familiar e as consequências negativas nos cuidados e na relação da díade mãe-bebê $\hat{e}^{22-25}$.

Os estudos que apontam para a relação significativa entre a ocorrência da depressão e alterações na relação entre a díade mãe-bebê sinalizam que os efeitos da depressão materna podem resultar negativamente no desenvolvimento da criança, potencializando desordens linguísticas, comportamentais, 
afetivas, cognitivas e sociais ${ }^{26}$. Nesse contexto, as síndromes depressivas que acometem mulheres nos primeiros meses após o parto afetam diretamente toda a família ${ }^{7}$.

Portanto, os achados deste estudo têm implicações importantes para o atendimento voltado à saúde mental de mulheres com morbidade materna grave e near miss, principalmente por ser pouco frequente a existência de registros na literatura brasileira sobre estratégias específicas para lidar com esse problema na atenção primária no contexto da saúde pública.

Algumas limitações devem ser consideradas nesta pesquisa, como o fato de não terem sido identificados na coleta dados específicos sobre os antecedentes de transtornos mentais anterior à gravidez e dados da história pessoal e familiar que pudessem influenciar nos aspectos psicológico e comportamental. Todavia, cabe ressaltar que o estudo mantém boa relevância científica, ao avaliar a relação entre a morbidade materna grave e near miss e a saúde mental das puérperas.

Tendo em vista a alta prevalência da depressão pós-parto no grupo de expostas à morbidade materna grave e near miss, os dados indicaram que a depressão causa vulnerabilidade psicossocial e afeta negativamente o estado emocional da mulher, ao mesmo tempo que reforça o seu significado como problema de saúde pública. Programas resultantes de políticas públicas, que visem a intervenções e estratégias de enfrentamento com equipe multiprofissional, por meio de instrumentos de rastreamento e identificação precoce dos sintomas, tornam-se importantes na rotina de protocolos de cuidado que favoreçam à atenção primária à saúde materna.

\section{REFERÊNCIAS}

1. Furuta M, Sandall J, Cooper D, Bick D. The relationship between severe maternal morbidity and psychological health symptoms at 6-8 weeks postpartum: a prospective cohort study in one English maternity unit. BMC Pregnancy Childbirth. 2014;14(133):1-14. PMid:24708797.

2. Iyengar K, Yadav R, Sen S. Consequences of maternal complications in women's lives in the first postpartum year: a prospective cohort study. J Health Popul Nutr. 2012;30(2):226-40. http://dx.doi.org/10.3329/jhpn. v30i2.11318. PMid:22838164

3. Geller SE, Rosenberg D, Cox SM, Kilpatrick S. Defining a conceptual framework for near miss maternal morbidity. J Am Med Womens Assoc. 2002;57(3):135-9. PMid:12146602.

4. Say L, Souza JP, Pattinson RC. WHO Working Group on Maternal Mortality and Morbidity Classifications. Maternal near miss-towards a standard tool for monitoring quality of maternal health care. Best Pract Res Clin Obstet Gynaecol. 2009;23(3):287-96. http://dx.doi.org/10.1016/j. bpobgyn.2009.01.007. PMid:19303368.

5. Silva DVR, Silveira MFA, Gomes-Sponholz FA. Experiences with severe maternal morbidity: a qualitative study on the perception of women. Rev Bras Enferm. 2016;69(4):618-24. PMid:27508470.

6. Koblinsky M, Chowdhury ME, Moran A, Ronsmans C. Maternal morbidity and disability and their consequences: neglected agenda in maternal health. J Health Popul Nutr. 2012;30(2):124-30. http://dx.doi.org/10.3329/jhpn. v30i2.11294. PMid:22838155.

7. World Health Organization. Mental health action plan 2013-2020 [online]. 2013 [citado em 2017 Mar 5]. Disponível em: http://www.who.int/ mental_health/action_plan_2013/en/

8. American Psychiatric Association. DSM-V. Manual Diagnóstico e Estatístico de Transtornos Mentais. $5^{\text {th }}$ ed. Porto Alegre: ARTMED; 2014.

9. Letourneau NL, Dennis CL, Benzies K, Duffett-Leger L, Stewart M, Tryphonopoulos PD, et al. Postpartum depression is a family affair: addressing the impact on mothers, fathers, and children. Issues Ment Health Nurs. 2012;33(7):445-57. http://dx.doi.org/10.3109/01612840.20 12.673054. PMid:22757597.

10. Norhayati MN, Nik Hazlina NH, Asrenee AR, Wan Emilin WMA. A Magnitude and risk factors for postpartum symptoms: a literature review. J

Affect Disord. 2015;175:34-52. http://dx.doi.org/10.1016/j.jad.2014.12.041. PMid:25590764.

11. Cox JL, Holden JM, Sagovsky R. Detection of postnatal depression: development of the 10-item Edinburgh Postnatal Depression Scale. Br J Psychiatry. 1987;150(6):782-6. http://dx.doi.org/10.1192/bjp.150.6.782. PMid:3651732.

12. Santos MFS, Martins FC, Pasquali L. Escala de auto-avaliação de depressão pós-parto: estudo no Brasil. Rev Psiquiatr Clin (Santiago). 1999;26(2):90-5.

13. Karlsen S, Say L, Souza JP, Hogue CJ, Calles DL, Gülmezoglu AM, et al. The relationship between maternal education and mortality among women giving birth in health care institutions: analysis of the cross sectional WHO Global Survey on Maternal and Perinatal Health. BMC Public Health. 2011;11:606. http://dx.doi.org/10.1186/1471-2458-11-606. PMid:21801399.

14. Rosendo TMSS, Roncalli AG. Prevalence and factors associated with Maternal Near Misses: a survey of the population in a capital city of the Brazilian Northeast. Ciênc. Saúde Coletiva. 2015;20(4):1295-304. http:// dx.doi.org/10.1590/1413-81232015204.09052014. PMid:25923639.

15. Adeoye IA, Onayade AA, Fatusi AO. Incidence, determinants and perinatal outcomes of near miss maternal morbidity in Ile-Ife Nigeria: a prospective case control study. BMC Pregnancy Childbirth. 2013;13(1):93. http://dx.doi. org/10.1186/1471-2393-13-93. PMid:23587107.

16. Pacheco AJC, Katz L, Souza RSA, Amorim MMR. Factors associated with severe maternal morbidity and near miss in the São Francisco Valley, Brazil: a retrospective, cohort study. BMC Pregnancy Childbirth. 2014;14(1):91. http://dx.doi.org/10.1186/1471-2393-14-91. PMid:24576223.

17. Filippi V, Ganaba R, Baggaley RF, Marshall T, Storeng KT, Sombié I, et al. Health of women after severe obstetric complications in Burkina Faso: a longitudinal study. Lancet. 2007;370(9595):1329-37. http://dx.doi. org/10.1016/S0140-6736(07)61574-8. PMid:17933647.

18. Sawyer A, Ayers S, Smith H. Pre- and postnatal psychological wellbeing in Africa: a systematic review. J Affect Disord. 2010;123(1-3):17-29. http:// dx.doi.org/10.1016/j.jad.2009.06.027. PMid:19635636.

19. Burgut FT, Bener A, Ghuloum S, Sheikh J. A study of postpartum depression and maternal risk factors in Qatar. J Psychosom Obstet Gynaecol. 2013;34(2):18. http://dx.doi.org/10.3109/0167482X.2013.786036. PMid:23701432. 
20. Mathisen SE, Glavin K, Lien L, Lagerløv P. Prevalence and risk factors for postpartum depressive symptoms in Argentina: a cross-sectional study. Int J Womens Health. 2013;5:787-93. http://dx.doi.org/10.2147/IJWH.S51436. PMid:24294009.

21. Norhayati MN, Nik Hazlina NH, Aniza AA, Asrenee AR. Severe Maternal Morbidity and Postpartum Depressive Symptomatology: a prospective double cohort comparison study. Res Nurs Health. 2016;39(6):415-25. http://dx.doi.org/10.1002/nur.21741. PMid:27367484.

22. Firoz T, Chou D, von Dadelszen P, Agrawal P, Vanderkruik R, Tunçalp $\mathrm{O}$, et al. Measuring maternal health: focus on maternal morbidity. Bull World Health Organ. 2013;91(10):794-6. http://dx.doi.org/10.2471/ BLT.13.117564. PMid:24115804.

23. Tunçalp O, Hindin MJ, Souza JP, Chou D, Say L. The prevalence of maternal near miss: a systematic review. BJOG. 2012;119(6):653-61. http://dx.doi. org/10.1111/j.1471-0528.2012.03294.x. PMid:22489760.
24. Storeng KT, Murray SF, Akoum MS, Ouattara F, Filippi V. Beyond body counts: a qualitative study of lives and loss in Burkina Faso after 'near-miss' obstetric complications. Soc Sci Med. 2010;71(10):1749-56. http://dx.doi. org/10.1016/j.socscimed.2010.03.056. PMid:20541307.

25. Assarag B, Dujardin B, Delamou A, Meski F-Z, De Brouwere V. Determinants of maternal near-miss in morocco: too late, too far, too sloppy? PLoS One. 2015;10(1):e0116675. http://dx.doi.org/10.1371/journal.pone.0116675. PMid:25612095.

26. Ikeda M, Hayashi M, Kamibeppu K. The relationship between attachment style and postpartum depression. Attach Hum Dev. 2014;16(6):557-72. http://dx.doi.org/10.1080/14616734.2014.941884. PMid:25098625.

Recebido em: Mar. 08, 2018 Aprovado em: Ago. 16, 2018 